Research Paper

\title{
Association of Body Mass Index Changes during Neoadjuvant Chemotherapy with Pathologic Complete Response and Clinical Outcomes in Patients with Locally Advanced Breast Cancer
}

\author{
Takahiro Kogawa ${ }^{1}$, Tamer M. Fouad ${ }^{1}$, Caimiao Wei ${ }^{2}$, Hiroko Masuda ${ }^{1}$, Kazuharu Kai ${ }^{1}$, Takeo Fujiii ${ }^{1}$, Randa \\ El-Zein ${ }^{3}$, Mariana Chavez-MacGregor ${ }^{1}$, Jennifer K. Litton ${ }^{1}$, Abenaa Brewster ${ }^{1}$, Ricardo H. Alvarez ${ }^{1}$, Gabriel \\ N. Hortobagyi ${ }^{1}$, Vicente Valero ${ }^{1}$, Richard Theriault ${ }^{1}$, and Naoto T. Ueno ${ }^{\circledR}$ \\ 1. Department of Breast Medical Oncology, The University of Texas MD Anderson Cancer Center, Houston, TX, USA \\ 2. Department of Biostatistics, The University of Texas MD Anderson Cancer Center, Houston, TX, USA \\ 3. Department of Epidemiology, The University of Texas MD Anderson Cancer Center, Houston, TX, USA
}

$\triangle$ Corresponding author: Naoto T. Ueno, MD, PhD, FACP, Morgan Welch Inflammatory Breast Cancer Research Program and Clinic, Section of Translational Breast Cancer Research, Department of Breast Medical Oncology Unit 1354, The University of Texas MD Anderson Cancer Center, 1515 Holcombe Blvd., Houston, TX 77030. Phone: (713) 792-8754; Fax: (713) 794-4385; Email: nueno@mdanderson.org

( ) Ivyspring International Publisher. This is an open-access article distributed under the terms of the Creative Commons License (http://creativecommons.org/ licenses/by-nc-nd/3.0/). Reproduction is permitted for personal, noncommercial use, provided that the article is in whole, unmodified, and properly cited.

Received: 2014.09.18; Accepted: 2014.10.22; Published: 2015.01.22

\begin{abstract}
The purpose of this study was to determine the association between body mass index (BMI) measurements (baseline BMI and changes in BMI during neoadjuvant systemic treatment [NST]) and clinical efficacy (pathologic complete response [PCR] rate and survival outcomes) in locally advanced breast cancer (LABC). We hypothesized that high baseline BMI and increases in BMI during NST are associated with lower PCR rates and poorer clinical outcomes in LABC.

We retrospectively reviewed the medical records of 1002 patients, 204 with primary inflammatory breast cancer (IBC) and 798 with stage III non-IBC, who underwent standard NST and definitive surgery between November 1, 2006, and December 31, 2012.

The median follow-up time for the survivors was 19.6 months ( $0.4-67.8$ months). The $P C R$ rates of patients whose BMI increased or decreased were $23.2 \%$ and $18.1 \%$, respectively, $(p=0.048)$. The unadjusted overall survival (OS) was significantly better in the group with increased BMI $(p=0.006)$. However, increased BMI was not an independent predictor of $P C R$ and clinical outcomes (recurrence-free survival and OS) after adjusting for other clinical variables. In subset analyses, increased $\mathrm{BMI}$ as a continuous variable was an independent predictor of higher $\mathrm{PCR}$ rates in the normal BMl/underweight group (odds ratio $[\mathrm{OR}]=1.35,95 \%$ confidence interval $[\mathrm{Cl}]$ : 1.06-0.71, $p=0.015$ ). Increased BMI (BMI change $\geq 0$ vs. $<0$ ) was also an independent predictor of $p C R$ $(\mathrm{OR}=1.65,95 \% \mathrm{Cl}: 1.00-2.72, p=0.049)$ in the postmenopausal group.

Our results show that increasing BMI shows improved clinical outcome in terms of better $P C R$ rates in normal BMI/underweight group and in the postmenopausal group. These results contradict previously reported findings on the association between high BMI and poor clinical efficacy regarding PCR rate and survival outcomes in early-stage breast cancer. Thus, the role of BMI in breast cancer may depend on patients' clinical characteristics such as advanced stage.
\end{abstract}

Key words: Breast cancer, Inflammatory breast cancer, Body mass index, Body mass index change, Predictive factor, Pathological complete response 


\section{Introduction}

Obesity and overweight are generally considered poor prognostic factors in early-stage breast cancer [1-4], although reports of this association have been inconsistent [5]. The impact of the association between obesity and prognosis in breast cancer differs according to patient and tumor characteristics such as menopausal status and tumor subtype, respectively. Since previous studies have focused on operable early-stage breast cancer, little is known about the association between baseline body mass index (BMI) and prognosis in locally advanced breast cancer (LABC) treated with current standard treatment -e.g., treated with surgery, chemotherapy (i.e., anthracycline and taxane), and radiation therapy with trastuzumab and/or hormone therapy if indicated.

In addition, high BMI (overweight: 25-29.9 $\mathrm{kg} / \mathrm{m}^{2}$, obese: $30 \mathrm{~kg} / \mathrm{m}^{2}$ or greater) correlates with breast cancer incidence in postmenopausal women, but the trend is inconsistent in premenopausal breast cancer patients [6]. A recent review revealed a positive association between pre-cancer weight and/or weight at diagnosis and poor survival outcome in 17 of 26 studies [7]. Several studies have shown that, in addition to high BMI at diagnosis, an increase in body weight after surgery is a poor prognostic factor for overall-survival (OS) $[2,8-10]$. Furthermore, BMI is a significant predictive factor for lower pathologic complete response (pCR) rates with neoadjuvant systemic treatment (NST) [11, 12]. Hence, high BMI and increasing BMI are generally considered poor predictive and prognostic markers for long-term outcome.

Further, patients with inflammatory breast cancer (IBC) have been reported to have higher body weight and a poor prognosis [13]. Studies of LABC have shown that high $\mathrm{BMI}$ is a poor prognostic factor. These findings may be partly explained by the observation that overweight and obese breast cancer patients tend to present with more advanced disease stage and have worse survival outcome compared to normal/underweight $\left(<25 \mathrm{~kg} / \mathrm{m}^{2}\right)$ patients [14]. These findings may also be explained by higher estrogen and androgen concentrations, especially in postmenopausal obese/overweight breast cancer patients [15, 16].

It is unclear whether an increase in BMI during NST truly predicts cancer outcomes or response to chemotherapy. Previous studies surveyed BMI and body weight changes for relatively longer periods than the duration of NST; therefore, knowledge about changes in BMI specifically during NST is scarce. Hence, we obtained these data during the short NST duration to determine the effect of BMI and BMI change on response to NST.
We hypothesized that high BMI at diagnosis and increases in BMI during NST would be associated with lower rates of $\mathrm{pCR}$ and poorer long-term clinical outcomes in LABC. The purpose of this study was to determine whether high baseline BMI or an increase in BMI from baseline to the completion of standard NST affects $\mathrm{pCR}$ and survival outcomes in LABC patients.

\section{Patients and Methods}

\section{Patient Selection}

This was a retrospective single-center investigation (protocol number: PA13-0131). Patients were identified from the Breast Cancer Management System database (protocol number: 2004-0541) at The University of Texas MD Anderson Cancer Center. Patients diagnosed with either IBC $(n=204)$ or stage III non-IBC $(\mathrm{n}=798)$ between November 1, 2006, and December 31, 2012 were included. All patients received standard NST consisting of anthracyclines and/or taxanes with or without concurrent trastuzumab followed by definitive surgery. IBC was defined as inflammatory changes in the involved breast, including diffuse erythema and edema (i.e., peau d'orange) involving the majority of the breast, with or without an underlying palpable mass involving the majority of the skin of the breast. Pathologic evidence of dermal lymphatic invasion was noted but not required for the diagnosis of IBC. Presumably, each case of IBC was diagnosed via a multidisciplinary team of investigators that included medical oncologists, surgeons, and radiologists as is standard at our institution.

The database included detailed information on patient demographics (e.g., age, sex, race, menopausal status), clinical characteristics (e.g., BMI at breast cancer diagnosis and at the conclusion of chemotherapy, treatment regimens and pathologic response in the breast and axilla), and tumor characteristics at diagnosis (e.g., clinical stage, estrogen receptor [ER], progesterone receptor [PR], and human epidermal growth factor receptor -2/ neu [HER2] status, and histologic grade). Follow-up information for patients whose data are included in the Breast Cancer Management System database is obtained every 2 years via direct review of the medical records and a direct link to the MD Anderson Tumor Registry, which mails annual follow-up letters to patients to determine their vital status and disease status. The Tumor Registry queries the Social Security Death Index and the Texas Bureau of Vital Statistics to determine the status of patients who do not respond to the letters. We collected all the above information and reconfirmed each patient's vital status and presence or ab- 
sence of recurrent disease based on chart review. This study protocol was approved by the MD Anderson Institutional Review Board.

\section{Measurement of BMI}

BMI was measured at diagnosis before chemotherapy and again at the last cycle of NST, and the change in BMI was calculated as the difference between the two. The value of BMI change was assessed as both a continuous variable and arbitrary categorical variable $(\geq 0$ vs. $<0)$ for this study.

We confirmed that candidates had not undergone definitive breast surgery prior to NST. Since BMI is a time-dependent variable, changes in BMI would be greater in patients who underwent NST for longer durations; therefore, to control bias, we excluded 6 patients who underwent NST for longer than 1 year (the regimen was unknown in 2 patients and delayed in 4 patients owing to adverse events) in the selection process.

\section{Pathology}

All pathologic evaluations were performed by breast cancer pathologists. $\mathrm{PCR}$ was defined as having no residual invasive carcinoma in the breast and no tumor in the axillary lymph nodes [17].

Immunohistochemical assays (IHC) were performed on formalin-fixed paraffin-embedded tissue sections from core needle biopsy samples to determine ER, PR, and HER2 status. Positive ER and PR status was defined as at least $10 \%$ of tumor cells with nuclear staining [18], and positive HR status was defined as either ER or PR positive. Typically, positive ER and PR status is defined as 1\% of tumor cells with nuclear staining; however, the nature of chart review precludes the use of a cutoff lower than $10 \%$.

Tumors were considered HER2 positive with a score of $3+$ on IHC and/or a fluorescence in situ hybridization ratio of greater than 2.0 [19].

\section{Treatment}

The cytotoxic agents administered for NST during the study period always included an anthracycline and/or taxane. These combination and single-agent regimens were administered with or without additional agents during the period evaluated in this study. Anthracycline-based regimens consisted of 4 courses of 5 -fluorouracil (5FU) at $500 \mathrm{mg} / \mathrm{m}^{2}$, epirubicin at $100 \mathrm{mg} / \mathrm{m}^{2}$, and cyclophosphamide 500 $\mathrm{mg} / \mathrm{m}^{2}$ (FEC) every 3 weeks; or $5 \mathrm{FU}$ at $500 \mathrm{mg} / \mathrm{m}^{2}$, doxorubicin at $50 \mathrm{mg} / \mathrm{m}^{2}$, and cyclophosphamide at $500 \mathrm{mg} / \mathrm{m}^{2}$ (FAC); or the same doses of epirubicin and cyclophosphamide (EC); or doxorubicin and cyclophosphamide (AC) given intravenously every 3 weeks. Taxane-based regimens most commonly consisted of 12 courses of weekly paclitaxel at $80 \mathrm{mg} / \mathrm{m}^{2}$.
Regimens of docetaxel at $100 \mathrm{mg} / \mathrm{m}^{2}$ or paclitaxel at $225 \mathrm{mg} / \mathrm{m}^{2}$ administered via 24-hour continuous intravenous (IV) infusion every 3 weeks were also used. When tumors were HER2 positive, trastuzumab was administered at $4 \mathrm{mg} / \mathrm{kg}$ IV over 90 minutes on the first day of treatment. Subsequent weekly doses of trastuzumab were administered at $2 \mathrm{mg} / \mathrm{kg}$ over 30 minutes. Trastuzumab was administered concurrently with FEC regimen reducing epirubicin to 75 $\mathrm{mg} / \mathrm{m}^{2}$. The other primary regimen for HER2+ breast cancer consisted of 6 cycles of docetaxel at $75 \mathrm{mg} / \mathrm{m}^{2}$ IV, carboplatin at an area under the concentration-time curve of $6 \mathrm{IV}$, and trastuzumab at $8 \mathrm{mg} / \mathrm{kg}$ IV on the first day followed by a $6 \mathrm{mg} / \mathrm{kg}$ maintenance dose every 3 weeks. Other regimens included anthracycline and taxane administered concurrently. Median NST duration was 215 days (range 48-364 days).

Patients who underwent 3 or more differing consecutive NST regimens were excluded owing to non-standard treatment.

\section{Statistical Methods}

The primary objectives were to assess the associations between baseline BMI and change in BMI during NST with pCR rates, recurrence-free survival (RFS) and OS. We also sought to identify associations between BMI and patient/tumor characteristics in each BMI category and determine whether IBC status and menopausal status affect $\mathrm{pCR}$ rates and survival outcomes.

RFS was defined as the time from definitive surgery until locoregional recurrence, the development of distant metastases, or death without a prior cancer event. In the absence of one of these events, RFS was censored at the last follow-up date. OS was defined as the time from definitive surgery until death from any cause or was censored at the date the patient was last known to be alive.

Continuous variables were summarized using medians (range), and categorical variables were summarized in frequency tables. Chi-squared tests were used to assess the bivariate associations between categorical variables. The Wilcoxon rank-sum test was used to assess the associations between pairs of continuous and binary variables.

The unadjusted distribution of OS and RFS was evaluated using the Kaplan-Meier method, and the differences in the distributions among groups were compared using the log-rank test. Cox proportional hazard models were used to assess the associations between BMI measures and survival outcomes with adjustments for IBC, HR, HER2, clinical stage, and lymphovascular invasion.

Logistic regression models were used to assess 
the association between BMI measures and pCR with adjustment for IBC, tumor HR and HER2 status, clinical stage, and presence or absence of lymphovascular invasion. A p-value less than 0.05 of each comparison was defined as significant.

\section{Results}

\section{Relationship between BMI and Patient and Tumor Characteristics}

The median follow-up time for survivors was 19.6 months (0.4-67.8 months). Of 204 patients with IBC and 798 patients with non-IBC, $125(61.3 \%)$ and $531(66.9 \%)$, respectively, had HR-positive tumors, and $54(26.5 \%)$ and $186(23.3 \%)$, respectively, had HER2-positive tumors. A total of 119 (58.3\%) patients with IBC and $420(52.9 \%)$ patients with non-IBC were postmenopausal. Overall, $33(16.2 \%)$ patients with IBC and $172(21.7 \%)$ with non-IBC achieved pCR with
NST. Tumor histology, nuclear grade, HR status, HER2 status, tumor stage (T stage) and lymph node involvement ( $\mathrm{N}$ stage) at diagnosis, and the NST agents administered were not significantly different among the three baseline BMI categories (Table 1).

The median BMI both at baseline and at the last cycle of chemotherapy was $28.8 \mathrm{~kg} / \mathrm{cm}^{2}$. On the basis of the aforementioned BMI categories, $73.7 \%$ of patients were overweight or obese. The proportion of African Americans in the overweight and obese groups was higher than that of other ethnic groups $(p$ $<0.001)$. Postmenopausal status $(p<0.001)$ and IBC ( $p$ $=0.015)$ were both significantly associated with obesity. In addition, the median age was lowest in the normal/underweight BMI group (46.0 years) compared to overweight (51.0 years) and obese (52.0 years, $p<0.001)$.

Table 1. Patients' characteristics among three baseline BMI categories

\begin{tabular}{|c|c|c|c|c|c|c|}
\hline \multirow[t]{3}{*}{ Characteristics } & \multirow[t]{3}{*}{ Levels } & Normal & Overweight & Obese & Total & \multirow[t]{3}{*}{$p$-value (chisquare) } \\
\hline & & BMI <25 & $25 \leq \mathrm{BMI}<30$ & BMI $\geq 30$ & $\mathrm{~N}=1002$ & \\
\hline & & $\mathrm{N}=\mathbf{2 6 3}$ & $\mathrm{N}=318$ & $\mathrm{~N}=421$ & & \\
\hline \multirow[t]{4}{*}{ Race } & White & $186(70.7 \%)$ & $182(57.2 \%)$ & $242(57.5 \%)$ & $610(60.9 \%)$ & \multirow[t]{4}{*}{$<0.001$} \\
\hline & Black & $14(5.3 \%)$ & $51(16.0 \%)$ & $94(22.3 \%)$ & $159(15.9 \%)$ & \\
\hline & Hispanic & $37(14.1 \%)$ & $66(20.8 \%)$ & $64(15.2 \%)$ & $167(16.7 \%)$ & \\
\hline & Others & $26(9.9 \%)$ & $19(6.0 \%)$ & $21(5.0 \%)$ & $66(6.6 \%)$ & \\
\hline \multirow[t]{2}{*}{ Menopause } & Post & $107(40.7 \%)$ & $169(53.5 \%)$ & $263(62.8 \%)$ & $539(54.0 \%)$ & \multirow[t]{2}{*}{$<0.001$} \\
\hline & Pre & $156(59.3 \%)$ & $147(46.5 \%)$ & $156(37.2 \%)$ & $459(46.0 \%)$ & \\
\hline \multirow[t]{3}{*}{ HISTOLOGY } & Ductal & $231(87.8 \%)$ & $288(90.6 \%)$ & $377(89.5 \%)$ & $896(89.4 \%)$ & \multirow[t]{3}{*}{0.657} \\
\hline & Lobular & $19(7.2 \%)$ & $21(6.6 \%)$ & $25(5.9 \%)$ & $65(6.5 \%)$ & \\
\hline & Others & $13(4.9 \%)$ & $9(2.8 \%)$ & $19(4.5 \%)$ & $41(4.1 \%)$ & \\
\hline \multirow[t]{2}{*}{ ER } & Negative & $93(35.4 \%)$ & $115(36.3 \%)$ & $155(37.0 \%)$ & $363(36.3 \%)$ & \multirow[t]{2}{*}{0.911} \\
\hline & Positive & $170(64.6 \%)$ & $202(63.7 \%)$ & $264(63.0 \%)$ & $636(63.7 \%)$ & \\
\hline \multirow[t]{2}{*}{ PR } & Negative & $143(54.8 \%)$ & $167(53.0 \%)$ & $231(55.3 \%)$ & $541(54.4 \%)$ & \multirow[t]{2}{*}{0.825} \\
\hline & Positive & $118(45.2 \%)$ & $148(47.0 \%)$ & $187(44.7 \%)$ & $453(45.6 \%)$ & \\
\hline \multirow[t]{3}{*}{ Nuclear grade } & I & $8(3.2 \%)$ & $9(2.9 \%)$ & $11(2.7 \%)$ & $28(2.9 \%)$ & \multirow[t]{3}{*}{0.538} \\
\hline & II & $69(27.6 \%)$ & $90(29.3 \%)$ & $96(23.8 \%)$ & $255(26.6 \%)$ & \\
\hline & III & $173(69.2 \%)$ & $208(67.8 \%)$ & $296(73.4 \%)$ & $677(70.5 \%)$ & \\
\hline \multirow[t]{2}{*}{ HER2 } & Negative & $202(76.8 \%)$ & $238(74.8 \%)$ & $322(76.5 \%)$ & $762(76.0 \%)$ & \multirow[t]{2}{*}{0.827} \\
\hline & Positive & $61(23.2 \%)$ & $80(25.2 \%)$ & $99(23.5 \%)$ & $240(24.0 \%)$ & \\
\hline \multirow[t]{2}{*}{ IBC } & No & $218(82.9 \%)$ & $263(82.7 \%)$ & $317(75.3 \%)$ & $798(79.6 \%)$ & \multirow[t]{2}{*}{0.015} \\
\hline & Yes & $45(17.1 \%)$ & $55(17.3 \%)$ & $104(24.7 \%)$ & $204(20.4 \%)$ & \\
\hline \multirow[t]{2}{*}{ LVI } & Negative & $154(59.0 \%)$ & $197(62.1 \%)$ & $259(61.7 \%)$ & $610(61.1 \%)$ & \multirow[t]{2}{*}{0.71} \\
\hline & Positive & $107(41.0 \%)$ & $120(37.9 \%)$ & $161(38.3 \%)$ & $388(38.9 \%)$ & \\
\hline \multirow[t]{3}{*}{ clinical_stage } & III/IIIA & $77(29.3 \%)$ & $91(28.6 \%)$ & $130(30.9 \%)$ & $298(29.7 \%)$ & \multirow[t]{3}{*}{0.059} \\
\hline & IIIB & $55(20.9 \%)$ & $80(25.2 \%)$ & $124(29.5 \%)$ & $259(25.8 \%)$ & \\
\hline & IIIC & $131(49.8 \%)$ & $147(46.2 \%)$ & $167(39.7 \%)$ & $445(44.4 \%)$ & \\
\hline \multirow[t]{4}{*}{ cT stage } & $\mathrm{T} 1$ & $12(4.6 \%)$ & $18(5.7 \%)$ & $11(2.6 \%)$ & $41(4.1 \%)$ & 0.192 \\
\hline & $\mathrm{T} 2$ & $64(24.7 \%)$ & $69(21.8 \%)$ & $91(21.7 \%)$ & $224(22.5 \%)$ & \\
\hline & T3 & $91(35.1 \%)$ & $103(32.5 \%)$ & $132(31.5 \%)$ & $326(32.8 \%)$ & \\
\hline & $\mathrm{T} 4$ & $92(35.5 \%)$ & $127(40.1 \%)$ & $185(44.2 \%)$ & $404(40.6 \%)$ & \\
\hline cN stage & N0 & $14(5.3 \%)$ & $23(7.2 \%)$ & $20(4.8 \%)$ & $57(5.7 \%)$ & 0.09 \\
\hline & N1 & $89(33.8 \%)$ & $112(35.2 \%)$ & $170(40.4 \%)$ & $371(37.0 \%)$ & \\
\hline & N2 & $28(10.6 \%)$ & $36(11.3 \%)$ & $62(14.7 \%)$ & $126(12.6 \%)$ & \\
\hline & N3 & $132(50.2 \%)$ & $147(46.2 \%)$ & $169(40.1 \%)$ & $448(44.7 \%)$ & \\
\hline BMI change & $<0$ & $105(39.9 \%)$ & $152(47.8 \%)$ & $265(62.9 \%)$ & $522(52.1 \%)$ & $<0.001$ \\
\hline & $\geq 0$ & $158(60.1 \%)$ & $166(52.2 \%)$ & $156(37.1 \%)$ & $480(47.9 \%)$ & \\
\hline Regimen & A & $5(1.9 \%)$ & $6(1.9 \%)$ & $8(1.9 \%)$ & $19(1.9 \%)$ & 0.365 \\
\hline & $\mathrm{AT}$ & 187(71.1\%) & $219(68.9 \%)$ & $298(70.8 \%)$ & $704(70.3 \%)$ & \\
\hline & ATH & $61(23.2 \%)$ & $67(21.1 \%)$ & $82(19.5 \%)$ & $210(21.0 \%)$ & \\
\hline & $\mathrm{T}$ & $7(2.7 \%)$ & $9(2.8 \%)$ & $13(3.1 \%)$ & $29(2.9 \%)$ & \\
\hline & $\mathrm{TH}$ & $3(1.1 \%)$ & $17(5.3 \%)$ & $20(4.8 \%)$ & $40(4.0 \%)$ & \\
\hline
\end{tabular}




\section{Associations between Changes in BMI and pCR to NST}

NST duration sometimes extends owing to adverse events. BMI change values were not significantly different among patients who underwent NST that took for less than 6 months, 6-9 months, or 9-12 months. In addition, differences in BMI changes were not significant based on the defined times as continuous variables.

The median BMI change in the entire study population was $-0.1 \mathrm{~kg} / \mathrm{cm}^{2}$ and was significantly higher in the patients who achieved pCR $\left(0.1 \mathrm{~kg} / \mathrm{cm}^{2}\right.$, (range -8.4, 6.1)) compared to patients who did not achieve pCR $\left(-0.1 \mathrm{~kg} / \mathrm{cm}^{2}\right.$, (range $\left.\left.-9.5,11.5\right) ; p=0.04\right)$. The pCR rate of the patients whose BMI increased $(23.2 \%)$ was significantly higher than that of the patients whose BMI decreased (18.1\%; $p=0.048)$. After adjusted for other clinical variables, increased BMI $(\geq$ 0 vs. $<0)$ was not shown to be a significant predictor of pCR in the overall group of patients $(p=0.225)$.

Without adjusted for other clinical variables, increased BMI was associated with a higher PCR rate in the normal/underweight group. This association was shown both through continuous variables $(p=0.034)$ and dichotomized categories of BMI change $\geq 0(p=$ 0.016). Increased BMI as a continuous variable independently predicted pCR $(p=0.015)$ in the nor$\mathrm{mal} /$ underweight group after adjusted for other clinical variables, however no such association was shown in the overweight and obese groups (Table 2).

\section{Associations between Changes in BMI and Survival Outcomes (RFS and OS)}

Increased BMI as a continuous variable was marginally associated with poor RFS in overweight patients $(p=0.082)$ but not in the normal/underweight and obese groups by multivariate analysis ( $p=0.633$ and $p=0.3887$, respectively) (Table 3).

As a categorical variable, increased BMI (BMI change $\geq 0$ vs. $<0$ ) predicted OS in all patients $(p=0.006$, Figure 1). After adjusting for other clinical variables, increased BMI did not predict OS. In all three BMI categories, increased BMI was not a prognostic indicator for OS (Table 4).

Table 2. Logistic regression analysis for $\mathrm{pCR}$ among three baseline $\mathrm{BMI}$ categories

\begin{tabular}{|c|c|c|c|c|c|}
\hline Parameter & & Odds ratio & $95 \% \mathrm{CI}$ & & Chi-square $p$-value \\
\hline \multicolumn{6}{|c|}{ A. Logistic regression analysis for pCR within BMI $<25(n=260)$} \\
\hline BMI change as continuous variable & & 1.345 & 1.059 & 1.709 & 0.0151 \\
\hline BMI (baseline) & & 1.054 & 0.87 & 1.276 & 0.5935 \\
\hline IBC & Yes vs. No & 0.308 & 0.09 & 1.05 & 0.0598 \\
\hline HR & POS vs. NEG & 0.185 & 0.09 & 0.383 & $<.0001$ \\
\hline HER2 & POS vs. NEG & 5.052 & 2.339 & 10.912 & $<.0001$ \\
\hline \multirow[t]{2}{*}{ Clinical stage } & IIIB vs. III/IIIA & 1.02 & 0.308 & 3.375 & 0.9745 \\
\hline & IIIC vs. III/IIIA & 1.297 & 0.575 & 2.925 & 0.5315 \\
\hline Lymphovasucular invasion & POS vs. NEG & 0.153 & 0.065 & 0.363 & $<.0001$ \\
\hline \multicolumn{6}{|c|}{ B. Logistic regression analysis for $\mathrm{pCR}$ within $\mathrm{BMI} \geq 25,<30(\mathrm{n}=315)$} \\
\hline BMI change as continuous variable & & 0.955 & 0.806 & 1.132 & 0.5957 \\
\hline BMI (baseline) & & 1.045 & 0.832 & 1.314 & 0.7037 \\
\hline IBC & Yes vs. No & 0.572 & 0.212 & 1.541 & 0.269 \\
\hline HR & POS vs. NEG & 0.273 & 0.14 & 0.531 & 0.0001 \\
\hline HER2 & POS vs. NEG & 5.949 & 3.026 & 11.697 & $<.0001$ \\
\hline \multirow[t]{2}{*}{ Clinical stage } & IIIB vs. III/IIIA & 1.353 & 0.476 & 3.851 & 0.5706 \\
\hline & IIIC vs. III/IIIA & 2.153 & 0.944 & 4.91 & 0.0684 \\
\hline Lymphovasucular invasion & POS vs. NEG & 0.28 & 0.125 & 0.628 & 0.002 \\
\hline \multicolumn{6}{|c|}{ C. Logistic regression analysis for $p C R$ within $B M I ~ \geq 30(n=416$} \\
\hline BMI change as continuous variable & & 0.991 & 0.902 & 1.089 & 0.8574 \\
\hline BMI (baseline) & & 1.024 & 0.979 & 1.07 & 0.302 \\
\hline IBC & Yes vs. No & 1.402 & 0.643 & 3.057 & 0.3962 \\
\hline HR & POS vs. NEG & 0.458 & 0.264 & 0.794 & 0.0054 \\
\hline HER2 & POS vs. NEG & 5.433 & 3.096 & 9.534 & $<.0001$ \\
\hline \multirow[t]{2}{*}{ Clinical stage } & IIIB vs. III/IIIA & 1.147 & 0.488 & 2.698 & 0.7529 \\
\hline & IIIC vs. III/IIIA & 1.631 & 0.844 & 3.154 & 0.1458 \\
\hline Lymphovasucular invasion & POS vs. NEG & 0.189 & 0.09 & 0.396 & $<.0001$ \\
\hline
\end{tabular}


Table 3. Cox regression analysis for RFS among three baseline BMI categories

\begin{tabular}{|c|c|c|c|c|c|}
\hline \multirow{2}{*}{\multicolumn{2}{|c|}{$\begin{array}{l}\text { Parameter } \\
\text { A. Cox regression analysis for RFS with } B M I<25(n=259)\end{array}$}} & Hazard Ratio & \multicolumn{2}{|l|}{$95 \% \mathrm{CI}$} & \multirow[t]{2}{*}{ Chi-square $p$-value } \\
\hline & & & & & \\
\hline BMI change as continuous variable & & 0.968 & 0.847 & 1.106 & 0.6333 \\
\hline BMI (baseline) & & 0.953 & 0.84 & 1.082 & 0.4607 \\
\hline IBC & Yes vs. No & 2.836 & 1.503 & 5.35 & 0.0013 \\
\hline $\mathrm{HR}$ & POS vs. NEG & 0.416 & 0.255 & 0.679 & 0.0005 \\
\hline HER2 & POS vs. NEG & 0.211 & 0.095 & 0.472 & 0.0001 \\
\hline \multirow[t]{2}{*}{ Clinical stage } & IIIB vs. III/IIIA & 0.785 & 0.366 & 1.688 & 0.536 \\
\hline & IIIC vs. III/IIIA & 0.834 & 0.458 & 1.519 & 0.5534 \\
\hline Lymphovasucular invasion & POS vs. NEG & 1.4 & 0.857 & 2.286 & 0.1786 \\
\hline \multicolumn{6}{|c|}{ B. Cox regression analysis for RFS with $25 \leq B M I<30(n=314)$} \\
\hline BMI change as continuous variable & & 1.124 & 0.985 & 1.281 & 0.0823 \\
\hline BMI (baseline) & & 0.97 & 0.807 & 1.166 & 0.744 \\
\hline IBC & Yes vs. No & 2.343 & 1.193 & 4.601 & 0.0135 \\
\hline $\mathrm{HR}$ & POS vs. NEG & 0.587 & 0.351 & 0.98 & 0.0417 \\
\hline HER2 & POS vs. NEG & 0.097 & 0.034 & 0.277 & $<.0001$ \\
\hline \multirow[t]{2}{*}{ Clinical stage } & IIIB vs. III/IIIA & 0.673 & 0.29 & 1.56 & 0.3556 \\
\hline & IIIC vs. III/IIIA & 1.203 & 0.631 & 2.294 & 0.5741 \\
\hline Lymphovasucular invasion & POS vs. NEG & 2.744 & 1.628 & 4.625 & 0.0002 \\
\hline \multicolumn{6}{|c|}{ C. Cox regression analysis for RFS with BMI>30 $(n=418)$} \\
\hline BMI change as continuous variable & & 1.023 & 0.971 & 1.078 & 0.3887 \\
\hline BMI (baseline) & & 1.004 & 0.973 & 1.037 & 0.7879 \\
\hline IBC & Yes vs. No & 1.831 & 1.073 & 3.127 & 0.0266 \\
\hline HR & POS vs. NEG & 0.336 & 0.221 & 0.51 & $<.0001$ \\
\hline HER2 & POS vs. NEG & 0.205 & 0.102 & 0.41 & $<.0001$ \\
\hline \multirow[t]{2}{*}{ Clinical stage } & IIIB vs. III/IIIA & 1.114 & 0.576 & 2.154 & 0.7484 \\
\hline & IIIC vs. III/IIIA & 1.086 & 0.638 & 1.85 & 0.7612 \\
\hline Lymphovasucular invasion & POS vs. NEG & 1.945 & 1.28 & 2.956 & 0.0018 \\
\hline
\end{tabular}

Table 4. Cox regression analysis for OS among three baseline BMI categories

\begin{tabular}{|c|c|c|c|c|c|}
\hline \multicolumn{2}{|c|}{ Parameter } & Hazard Ratio & \multicolumn{2}{|l|}{$95 \% \mathrm{CI}$} & \multirow[t]{2}{*}{ Chi-square $p$-value } \\
\hline A. Cox regression analysis for OS & 261) & & & & \\
\hline BMI change as continuous variable & & 0.995 & 0.826 & 1.199 & 0.9578 \\
\hline BMI (baseline) & & 0.996 & 0.84 & 1.181 & 0.962 \\
\hline IBC & Yes vs. No & 1.974 & 0.821 & 4.744 & 0.1285 \\
\hline HR & POS vs. NEG & 0.269 & 0.139 & 0.522 & 0.0001 \\
\hline HER2 & POS vs. NEG & 0.212 & 0.074 & 0.609 & 0.0039 \\
\hline \multirow[t]{2}{*}{ Clinical stage } & IIIB vs. III/IIIA & 0.668 & 0.227 & 1.963 & 0.4627 \\
\hline & IIIC vs. III/IIIA & 0.889 & 0.41 & 1.924 & 0.7644 \\
\hline Lymphovasucular invasion & POS vs. NEG & 1.113 & 0.568 & 2.179 & 0.755 \\
\hline \multicolumn{6}{|c|}{ B. Cox regression analysis for OS with $25 \leq B M I<30(n=316)$} \\
\hline BMI change as continuous variable & & 1.041 & 0.88 & 1.233 & 0.6376 \\
\hline BMI (baseline) & & 0.833 & 0.652 & 1.065 & 0.1445 \\
\hline IBC & Yes vs. No & 1.498 & 0.624 & 3.592 & 0.3655 \\
\hline HR & POS vs. NEG & 0.697 & 0.348 & 1.395 & 0.3078 \\
\hline HER2 & POS vs. NEG & 0.22 & 0.08 & 0.603 & 0.0033 \\
\hline \multirow[t]{2}{*}{ Clinical stage } & IIIB vs. III/IIIA & 0.947 & 0.29 & 3.097 & 0.9281 \\
\hline & IIIC vs. III/IIIA & 1.888 & 0.752 & 4.741 & 0.1759 \\
\hline Lymphovasucular invasion & POS vs. NEG & 2.088 & 1.065 & 4.092 & 0.032 \\
\hline \multicolumn{6}{|c|}{ C. Cox regression analysis for OS with BMI $>30(n=418)$} \\
\hline BMI change as continuous variable & & 0.99 & 0.91 & 1.077 & 0.8119 \\
\hline BMI (baseline) & & 0.995 & 0.952 & 1.041 & 0.8352 \\
\hline IBC & Yes vs. No & 1.538 & 0.774 & 3.056 & 0.2196 \\
\hline HR & POS vs. NEG & 0.21 & 0.12 & 0.368 & $<.0001$ \\
\hline HER2 & POS vs. NEG & 0.032 & 0.004 & 0.233 & 0.0007 \\
\hline \multirow[t]{2}{*}{ Clinical stage } & IIIB vs. III/IIIA & 1.431 & 0.621 & 3.298 & 0.4002 \\
\hline & IIIC vs. III/IIIA & 1.038 & 0.518 & 2.08 & 0.9169 \\
\hline Lymphovasucular invasion & POS vs. NEG & 1.481 & 0.86 & 2.551 & 0.1565 \\
\hline
\end{tabular}



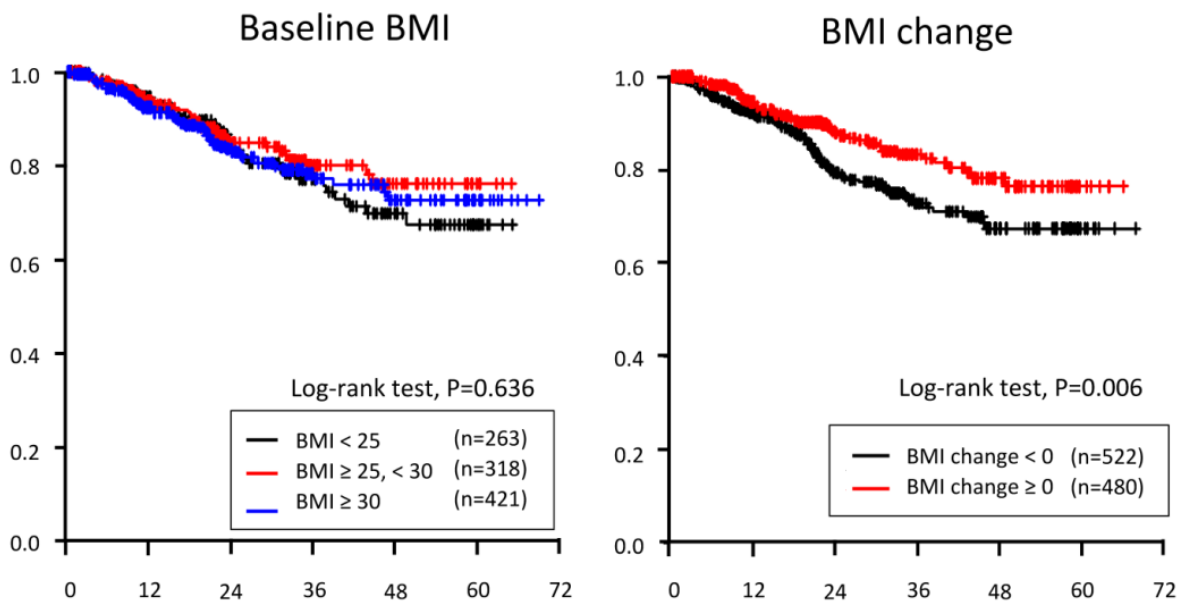

Figure 1. Kaplan-Merier curve of overall-survival based on three baseline BMI categories (left) and BMI change during neoadjuvant systemic treatment

\section{Associations between Baseline BMI and PCR/RFS/OS}

The median BMI among pCR and non-pCR groups was $28.7 \mathrm{~kg} / \mathrm{cm}^{2}$ (range 18.7-77.1) and 28.8 $\mathrm{kg} / \mathrm{cm}^{2}$ (range 16.7- 64.1), respectively $(p=0.64)$. The $\mathrm{pCR}$ rate in the three BMI categories (nor$\mathrm{mal} /$ underweight, overweight, and obese) was $24.0 \%$, $18.6 \%$, and $19.8 \%$, respectively $(p=0.243$ ) (Supplementary table 1 ).

Baseline BMI as a continuous variable was not significantly associated with RFS in univariate $(p=$ $0.70)$ or multivariate analysis $(p=0.57)$ in the overall cohort. Likewise, baseline BMI as a continuous variable was not associated with OS in univariate $(p=0.99)$ or multivariate analysis $(p=0.72)$ in the overall cohort.

Univariable analysis did not demonstrate any OS difference among the three baseline BMI categories $(p$ $=0.636$ ).

\section{Associations between BMI Changes and Breast Cancer Type}

The proportion of patients with IBC in the obese group $(24.7 \%)$ was significantly higher than that in the overweight (17.3\%) and normal/underweight $(17.1 \%)$ BMI categories $(p=0.015)$. BMI change $(\geq 0$ vs. $<0)$ did not predict the $\mathrm{pCR}$ rate in the IBC $(p=0.19)$ or non-IBC group $(p=0.14)$, and the baseline BMI category was not significantly associated with the $\mathrm{pCR}$ rate when analyzed separately in patients with IBC $(p$ $=0.55)$ and those with non-IBC $(p=0.1)$. The association of BMI change $(\geq 0$ vs. $<0)$ with RFS and OS was not significantly different among the three BMI categories in the non-IBC group; however, OS was significantly better in patients in the IBC group with a BMI change of $\geq 0$ ( $p<0.001)$. This result was supported by the finding that increased BMI as a continuous variable was associated with better OS $(p=0.037)$ in the IBC cohort. In multivariate analysis, BMI change did not predict $\mathrm{pCR}$ or survival outcome in either the IBC or non-IBC cohort.

\section{Association between BMI Changes and Men- opausal Status}

The proportion of postmenopausal patients was significantly higher in the obese group $(62.8 \%)$ than in the overweight $(53.5 \%)$ and normal/underweight groups $(40.7 \% ; p<0.001)$; however, the proportion of patients with decreased BMI was significantly higher in the obese group than in the overweight and normal/underweight groups $(p<0.001)$.

An increase in BMI was not a significant predictor of $\mathrm{pCR}$ in premenopausal patients; however, it was significantly associated with $\mathrm{pCR}$ in the postmenopausal cohort $(p=0.045)$ on univariate analysis. Increased BMI was also significantly associated with OS $(p=0.01)$. After adjustments for other clinical variables (BMI, IBC, HR, and HER2 status, clinical stage, and lymphovascular invasion), increased BMI remained to be an independent significant predictor of $\mathrm{pCR}(\mathrm{p}=0.05)$ but not of OS $(p=0.14)$.

\section{Discussion}

In this study, an increase in BMI during NST was a significant predictor of higher $\mathrm{pCR}$ rates in IBC and non-inflammatory LABC patients with normal or low $\mathrm{BMI}$ and was significantly associated with higher $\mathrm{pCR}$ in postmenopausal patients with LABC. However, due to the lack of extensive follow-up data, we cannot suggest that increasing weight is beneficial in this population. In addition, we estimated that some patients might have had chemotherapy resistance due to cachexia or sarcopenia with decreased body weight $[20,21]$, although this may occurs even in obese patients [22].

We hypothesized that increased body weight was associated with poor survival outcomes; howev- 
er, this was only a suggested tendency in overweight breast cancer patients in this study. Interestingly, our study included a relatively large number of patients with IBC, and a large proportion of these were in the obese group. Some biological aspects of IBC remain unclear, and the associations between BMI changes and IBC outcomes are not known. In our study, an increase in BMI during NST was partially associated with better IBC outcomes, and this might have had an effect on RFS in obese breast cancer patients.

Increased BMI was previously evaluated as a poor prognostic indicator in the adjuvant chemotherapy setting [2,9] and was associated with poor outcome with a relatively long evaluation period. These previous studies generally included hormone therapy (e.g., aromatase inhibitors), and many of the patients therein were postmenopausal and tended to gain weight during therapy. Therefore, the impact of increased BMI during chemotherapy was different in the previous studies.

The novelty of this study is that we tested the associations among BMI at diagnosis, $\mathrm{pCR}$ rates, and survival in LABC. In addition, this is the first study to determine whether increased BMI during NST is associated with $\mathrm{pCR}$ rates and survival. We focused on increased BMI during NST administered for approximately 6 months with current standard regimen. By including patients after trastuzumab was permitted to be used as NST, we analyzed BMI specifically in the context of the current standard treatment regimens. However, the chemotherapy doses sometimes have been capped in obese patients to prevent adverse events that were previously noted to be associated with poorer clinical outcomes [23]; we consistently administered standard chemotherapy doses based on patients' height and body weight.

One limitation of our study is its retrospective nature. Hence, we could not identify the adverse effects of chemotherapy or determine their grade, and we could not define the chemotherapy dose intensity. In addition, we could not determine whether normal/underweight BMI patients may have been ill from their cancers and therefore experienced beneficial effects of weight gain. We speculate that BMI changes could have been affected by changes in appetite due to chemotherapy-related adverse effects. Other factors such as tumor aggressiveness might have also affected BMI. Furthermore, we did not collect data about patients' lifestyles, such as smoking status, which might have affected the results, since smoking has the potential to minimize weight gain and attenuate mortality $[2,24]$.

Several studies have reported that weight gain is a general side effect in women receiving adjuvant chemotherapy for breast cancer [25-27], and chemo- therapy has been associated with greater weight gain than other adjuvant treatments such as tamoxifen or radiotherapy [28-30]. In this regard, chemotherapy may be the only intervention that caused increased BMI during NST since standard NST has not included hormone therapy and radiotherapy thus far.

Several factors could result in BMI changes during chemotherapy. Chemotherapy-induced nausea and appetite loss could result in decreased body weight, and depression following a breast cancer diagnosis could also have an effect. Concomitant steroid use, dietary recommendations, and reduced physical activity could lead to increases in body weight [31, 32]. In contrast, some patients may have cachexia owing to disease progression, or obese patients may reduce their $\mathrm{BMI}$ via a healthy diet and active exercise [33]; thus, we cannot generalize changes in BMI as a single predictive/prognostic indicator without broader lifestyle information.

With the aforementioned limitations in our study, increased BMI, but not baseline BMI, was associated with better pCR rates in LABC. Either neoadjuvant or adjuvant chemotherapy can be administered for early-stage breast cancer, but for the purposes of down-staging, NST is preferred for LABC. Other than baseline BMI, increased BMI is a possible predictive biomarker in LABC.

This study suggests the importance of $\mathrm{BMI}$ in the setting of LABC treated with NST, since few other BMI studies focused in this group. With previous BMI research results, we may have more precise strategies for delivering NST based on disease stage and baseline BMI. Medical practitioners may recommend lifestyle choices changes such as exercise and improved nutritional balance aimed at reducing body weight during breast cancer follow-up, however, these recommendations may be beneficial for only overweight or obese patients with early-stage breast cancer.

In addition, our results showed that increased BMI with NST was associated with a trend toward poorer RFS in overweight patients. Once body weight starts increasing, this change may continue for extended periods owing to chemotherapy-induced ovarian failure [34] which induces fat tissue accumulation [35]. Hence, we do not recommend as an objective increasing BMI during NST since large BMI changes may be difficult to control.

In summary, high BMI at baseline was not associated with $\mathrm{pCR}$ in IBC and non-inflammatory LABC patients treated with NST; however, an increase in BMI during NST was associated with a significantly better pCR rate, especially in normal/underweight and postmenopausal breast cancer patients. We speculate that baseline BMI might have played a minor role in determining treatment response and patient 
prognosis, whereas tumor biology is a more important prognostic factor in patients with aggressive breast cancer such as in locally advanced disease. Hence, the fact that increase in BMI was a significant predictor of $\mathrm{pCR}$ in the normal/underweight group or postmenopausal group implies it may be important to manage body weight during NST. The role of BMI in breast cancer may depend on disease stage and warrant further investigation.

The results of this study suggest that increasing BMI during chemotherapy is beneficial for patients with aggressive types of cancer, since aggressive tumors are more likely to induce cachexia. Hence, we hypothesize that the impact of BMI changes on chemotherapy response and survival outcome is different according to the breast cancer subtype. Further study is warranted to determine whether changes in BMI have predictive and prognostic value according to breast cancer subtype.

\section{Supplementary Material}

Supplementary Table 1.

http://www.jcancer.org/v06p0310s1.pdf

\section{Acknowledgement}

Markeda L. Wade of the Department of Scientific Publications at The University of Texas MD Anderson Cancer Center provided scientific editing services.

\section{Funding}

This study was funded by a My Oncology Dream award from the Japan Cancer Society, the Morgan Welch Inflammatory Breast Cancer Research Program, the National Institutes of Health through MD Anderson's Cancer Center Support Grant CA016672, and a grant from the State of Texas Rare and Aggressive Breast Cancer Research Program.

\section{Competing Interests}

The authors have declared that no competing interest exists.

\section{References}

1. Bastarrachea J, et al. Obesity as an adverse prognostic factor for patients receiving adjuvant chemotherapy for breast cancer. Ann Intern Med, 1994. 120(1): 18-25.

2. Kroenke C.H, et al. Weight, weight gain, and survival after breast cancer diagnosis. J Clin Oncol, 2005. 23(7): 1370-8.

3. Loi $S$, et al. Obesity and outcomes in premenopausal and postmenopausal breast cancer. Cancer Epidemiol Biomarkers Prev, 2005. 14(7): 1686-91.

4. Senie R.T, et al. Obesity at diagnosis of breast carcinoma influences duration of disease-free survival. Ann Intern Med, 1992. 116(1): 26-32.

5. Obermair A, et al. The influence of obesity on the disease-free survival in primary breast cancer. Anticancer Res, 1995. 15(5B): 2265-9.

6. Renehan A.G, et al. Body-mass index and incidence of cancer: a systematic review and meta-analysis of prospective observational studies. Lancet, 2008. 371(9612): 569-78.

7. Rock C.L and Demark-Wahnefried W. Nutrition and survival after the diagnosis of breast cancer: a review of the evidence. J Clin Oncol, 2002. 20(15): 3302-16.
8. Camoriano J.K, et al. Weight change in women treated with adjuvant therapy or observed following mastectomy for node-positive breast cancer. J Clin Oncol, 1990. 8(8): 1327-34.

9. Thivat $\mathrm{E}$, et al. Weight change during chemotherapy changes the prognosis in non metastatic breast cancer for the worse. BMC Cancer, 2010. 10: 648 .

10. Nichols H.B, et al. Body Mass Index Before and After Breast Cancer Diagnosis: Associations with All-Cause, Breast Cancer, and Cardiovascular Disease Mortality. Cancer Epidemiology Biomarkers \& Prevention, 2009. 18(5): 1403-1409.

11. Litton J.K, et al. Relationship between obesity and pathologic response to neoadjuvant chemotherapy among women with operable breast cancer. J Clin Oncol, 2008. 26(25): 4072-7.

12. Chen $\mathrm{S}$, et al. Obesity or overweight is associated with worse pathological response to neoadjuvant chemotherapy among Chinese women with breast cancer. PLoS One, 2012. 7(7): e41380.

13. Chang $S$, et al. Inflammatory breast cancer survival: the role of obesity and menopausal status at diagnosis. Breast Cancer Res Treat, 2000. 64(2): 157-63.

14. Cui $Y$, et al. Body mass and stage of breast cancer at diagnosis. Int J Cancer, 2002. 98(2): 279-83.

15. Chlebowski R.T., Aiello E, and McTiernan A. Weight loss in breast cancer patient management. J Clin Oncol, 2002. 20(4): 1128-43.

16. McTiernan A, et al. Adiposity and sex hormones in postmenopausal breast cancer survivors. J Clin Oncol, 2003. 21(10): 1961-6.

17. Cortazar $\mathrm{P}$, et al. Pathological complete response and long-term clinical benefit in breast cancer: the CTNeoBC pooled analysis. Lancet, 2014.

18. Elledge, R.M, et al. Estrogen receptor (ER) and progesterone receptor (PgR), by ligand-binding assay compared with ER, PgR and pS2, by immuno-histochemistry in predicting response to tamoxifen in metastatic breast cancer: a Southwest Oncology Group Study. Int J Cancer, 2000. 89(2): $111-7$.

19. [Internet] NCCN Clinical Practice Guidelines in Oncology (NCCN Guidelines) $\begin{array}{lll}\text { Breast Cancer } & \text { Version } & \end{array}$ http://www.nccn.org/professionals/physician_gls/pdf/breast.pdf.

20. Zhou X, et al. Reversal of cancer cachexia and muscle wasting by ActRIIB antagonism leads to prolonged survival. Cell, 2010. 142(4): 531-43.

21. Villasenor A, et al. Prevalence and prognostic effect of sarcopenia in breast cancer survivors: the HEAL Study. J Cancer Surviv, 2012. 6(4): 398-406.

22. Del Fabbro E, et al. The relationship between body composition and response to neoadjuvant chemotherapy in women with operable breast cancer. Oncologist, 2012. 17(10): 1240-5.

23. Wood W.C, et al. Dose and dose intensity of adjuvant chemotherapy for stage II, node-positive breast carcinoma. N Engl J Med, 1994. 330(18): 1253-9.

24. Manson J.E, et al. Body weight and mortality among women. N Engl J Med, 1995. 333(11): 677-85.

25. Gadea E, et al. Importance of metabolic changes induced by chemotherapy on prognosis of early-stage breast cancer patients: a review of potential mechanisms. Obes Rev, 2012. 13(4): 368-80.

26. Goodwin P.J, et al. Adjuvant treatment and onset of menopause predict weight gain after breast cancer diagnosis. J Clin Oncol, 1999. 17(1): 120-9.

27. Demark-Wahnefried W., Winer E.P, and Rimer B.K. Why women gain weight with adjuvant chemotherapy for breast cancer. J Clin Oncol, 1993. 11(7): 1418-29.

28. Chlebowski R.T, et al. Dietary fat reduction and breast cancer outcome: interim efficacy results from the Women's Intervention Nutrition Study. J Natl Cancer Inst, 2006. 98(24): 1767-76.

29. Heideman W.H, et al. The frequency, magnitude and timing of post-diagnosis body weight gain in Dutch breast cancer survivors. Eur J Cancer, 2009. 45(1): 119-26.

30. Saquib N, et al. Weight gain and recovery of pre-cancer weight after breast cancer treatments: evidence from the women's healthy eating and living (WHEL) study. Breast Cancer Res Treat, 2007. 105(2): 177-86.

31. Irwin M.L, et al. Physical activity levels before and after a diagnosis of breast carcinoma: the Health, Eating, Activity, and Lifestyle (HEAL) study. Cancer, 2003. 97(7): 1746-57.

32. Rock C.L, et al. Factors associated with weight gain in women after diagnosis of breast cancer. Women's Healthy Eating and Living Study Group. J Am Diet Assoc, 1999. 99(10): 1212-21.

33. Wilson D.B, et al. Anthropometric changes using a walking intervention in African American breast cancer survivors: a pilot study. Prev Chronic Dis, 2005. 2(2): A16.

34. Angelucci D, et al. Long-term outcome of neoadjuvant systemic therapy for locally advanced breast cancer in routine clinical practice. J Cancer Res Clin Oncol, 2013. 139(2): 269-80.

35. Gordon A.M, et al. Premature ovarian failure and body composition changes with adjuvant chemotherapy for breast cancer. Menopause, 2011. 18(11): 1244-8. 